ARTICLE

Received 2 Jun 2014 | Accepted 17 Sep 2014 | Published 20 Oct 2014

DOI: $10.1038 /$ ncomms 6290

\title{
Towards intrinsic charge transport in monolayer molybdenum disulfide by defect and interface engineering
}

\author{
Zhihao Yu${ }^{1, \star}$, Yiming Pan ${ }^{2, \star}$, Yuting Shen ${ }^{3}$, Zilu Wang ${ }^{4}$, Zhun-Yong Ong ${ }^{5}$, Tao $\mathrm{Xu}^{3}$, Run Xin ${ }^{1}$, Lijia Pan $^{1}$,
} Baigeng Wang ${ }^{2}$, Litao Sun ${ }^{3}$, Jinlan Wang ${ }^{4}$, Gang Zhang ${ }^{5}$, Yong Wei Zhang ${ }^{5}$, Yi Shi ${ }^{1} \&$ Xinran Wang ${ }^{1}$

Molybdenum disulfide is considered as one of the most promising two-dimensional semiconductors for electronic and optoelectronic device applications. So far, the charge transport in monolayer molybdenum disulfide is dominated by extrinsic factors such as charged impurities, structural defects and traps, leading to much lower mobility than the intrinsic limit. Here we develop a facile low-temperature thiol chemistry route to repair the sulfur vacancies and improve the interface, resulting in significant reduction of the charged impurities and traps. High mobility $>80 \mathrm{~cm}^{2} \mathrm{~V}^{-1} \mathrm{~s}^{-1}$ is achieved in backgated monolayer molybdenum disulfide field-effect transistors at room temperature. Furthermore, we develop a theoretical model to quantitatively extract the key microscopic quantities that control the transistor performances, including the density of charged impurities, short-range defects and traps. Our combined experimental and theoretical study provides a clear path towards intrinsic charge transport in two-dimensional dichalcogenides for future high-performance device applications.

\footnotetext{
${ }^{1}$ National Laboratory of Solid State Microstructures, School of Electronic Science and Engineering and Collaborative Innovation Center of Advanced Microstructures, Nanjing University, Nanjing 210093, China. ${ }^{2}$ School of Physics, Nanjing University, Nanjing 210093, China. ${ }^{3}$ SEU-FEl Nano-Pico Center, Key Laboratory of MEMS of Ministry of Education, Southeast University, Nanjing 210096, China. ${ }^{4}$ Department of Physics, Southeast University, Nanjing 211189, China. ${ }^{5}$ Institute of High Performance Computing, 1 Fusionopolis Way, Singapore 138632, Singapore. * These authors contributed equally to this work. Correspondence and requests for materials should be addressed to L.S. (email: slt@seu.edu.cn) or to Y.S. (email: yshi@nju.edu.cn) or to X.W. (email: xrwang@nju.edu.cn).
} 
D espite its great promise as a two-dimensional (2D) channel material for logic transistors ${ }^{1,2}$, integrated circuits $^{3,4}$ and photodetectors ${ }^{5,6}$, the charge transport in monolayer of molybdenum disulfide $\left(\mathrm{MoS}_{2}\right)$ is still far away from the intrinsic limit. Theoretically, the phonon-limited mobility is $\sim 410 \mathrm{~cm}^{2} \mathrm{~V}^{-1} \mathrm{~s}^{-1}$ at room temperature and is weakly dependent on carrier density until $\sim 10^{13} \mathrm{~cm}^{-2}$, where electron-electron scattering starts to play important roles $^{7}$. Experimentally, however, very different behaviour is observed regardless of the sample preparation method. First, the electron mobility in backgated monolayer $\mathrm{MoS}_{2}$ devices is limited to $\sim 40 \mathrm{~cm}^{2} \mathrm{~V}^{-1} \mathrm{~s}^{-1}$ at room temperature, an order of magnitude lower than the phonon limit ${ }^{2,8-10}$. Second, the mobility is found to increase with carrier density even beyond $10^{13} \mathrm{~cm}^{-2}$ (refs 2,9). Third, at low carrier density, the charge transport is dominated by hopping mechanism ${ }^{11,12}$. Fourth, a metal-insulator transition (MIT) is observed at high carrier densities on the order of $10^{13} \mathrm{~cm}^{-2}$, but the underlying mechanism remains debated ${ }^{2,8,9}$. These observations clearly point to the existence of extrinsic factors that dominate charge transport in monolayer $\mathrm{MoS}_{2}$. Charged impurities $(\mathrm{CI})^{2,13}$, short-range defects ${ }^{11}$ and localized states $^{11,14,15}$, among others, are believed to strongly influence electron transport. However, a comprehensive physical picture that explains all of above-mentioned transport phenomena and provides quantitative and microscopic insights into the impurities is still lacking.

Owing to its atomic thickness, electrons in monolayer $\mathrm{MoS}_{2}$ are more susceptible to impurities both inside $\mathrm{MoS}_{2}$ and at the dielectric interface. Therefore, the engineering of defects and interface represents a logical route to further improve $\mathrm{MoS}_{2}$ device performance. Recently, the use of thiol-terminated $\mathrm{SiO}_{2}$ (ref. 16), boron nitride ${ }^{17}$ and suspended structure ${ }^{18}$ were found to improve the mobility of monolayer $\mathrm{MoS}_{2}$ devices by several folds, showing the importance of interface. However, their mobility values are still much lower than the intrinsic limit, indicating that other sources of impurities, most likely inside $\mathrm{MoS}_{2}$, are still present.

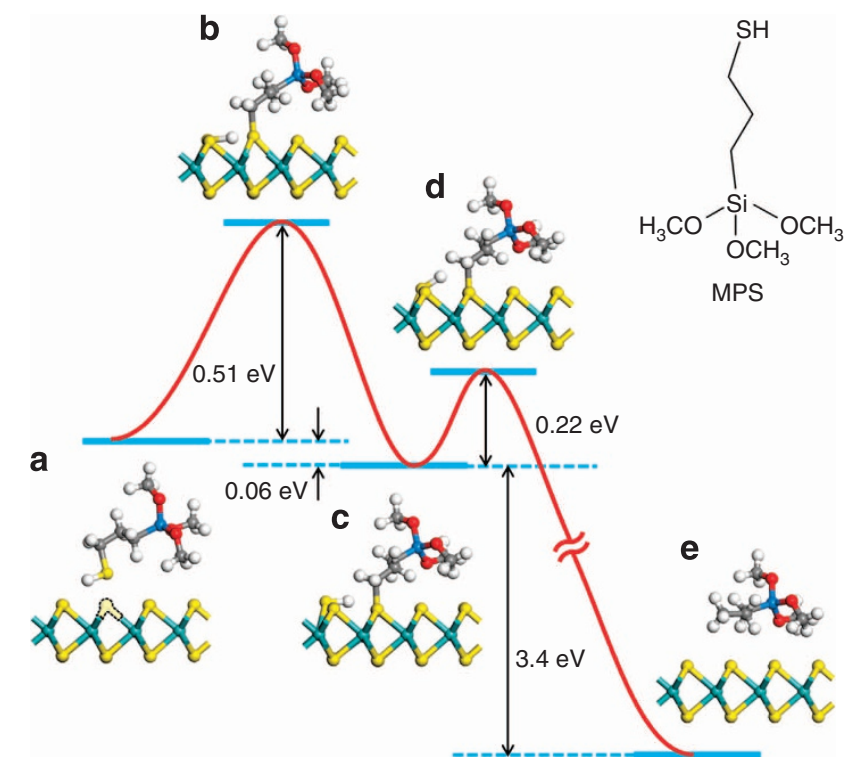

Figure 1 | Kinetics and transient states of the reaction between a

single SV and MPS. There are two energy barriers, the first one $(0.51 \mathrm{eV})$ is due to the $\mathrm{S}-\mathrm{H}$ bond breaking, and the second one $(0.22 \mathrm{eV})$ is due to $\mathrm{S}-\mathrm{C}$ bond breaking. (a-e) Plots the initial, transient and final states of the reaction. The SV in the initial state is illustrated by dashed line in (a). The inset shows the chemical structure of MPS.
In this work, we show that sulfur vacancies (SVs), which are the main type of intrinsic defects in $\mathrm{MoS}_{2}$ (ref. 11), can be effectively repaired by (3-mercaptopropyl)trimethoxysilane (MPS) under mild annealing, resulting in significant reduction of CI and short-range scattering. Monolayer $\mathrm{MoS}_{2}$ with both sides treated by MPS exhibits a record-high mobility of $81 \mathrm{~cm}^{2} \mathrm{~V}^{-1} \mathrm{~s}^{-1}\left(320 \mathrm{~cm}^{2} \mathrm{~V}^{-1} \mathrm{~s}^{-1}\right)$ at room temperature (low temperature), much higher than untreated samples. In addition, we show that MIT in $\mathrm{MoS}_{2}$ is due to localized trap states, which can be modulated by improving the sample and interface quality. A theoretical model that takes into account the major scattering sources (phonon, CI and short-range defects) and localized charge traps is developed to quantitatively understand the scaling of mobility, conductivity and MIT in monolayer $\mathrm{MoS}_{2}$. By fitting the experimental data, we are able to extract key microscopic quantities including the density of $\mathrm{CI}$, short-range defects and charge traps, as well as derive a transport phase diagram for $\mathrm{MoS}_{2}$. The quantitative information also allows us to discuss the possible origins of these extrinsic factors, which serve as the basis for further device optimization.

\section{Results}

Repair of SVs by thiol chemistry. The monolayer $\mathrm{MoS}_{2}$ samples studied here are obtained by mechanical exfoliation from bulk crystals (Supplementary Fig. 1). A high density of SV exists in asexfoliated $\mathrm{MoS}_{2}$ as demonstrated in earlier works ${ }^{11,19}$. These defects, which can act as catalytic sites for hydrodesulfurization reaction $s^{20,21}$, are chemically reactive. Therefore, it is possible to repair the SV by thiol chemistry. Here, we choose a specific molecule MPS (Fig. 1 inset) for two reasons. (i) The S-C bond in MPS is weaker than other thiol molecules like dodecanethiol due to the acidic nature of $\mathrm{CH}_{3}-\mathrm{O}$ - groups, leading to a low energy barrier for the reaction 22 . (ii) The trimethoxysilane groups in MPS react with the $\mathrm{SiO}_{2}$ substrate to form a self-assembled monolayer $^{23}$ (SAM, Supplementary Fig. 2). The SAM layer can effectively passivate the $\mathrm{MoS}_{2} / \mathrm{SiO}_{2}$ interface, while the outstanding thiol group can also repair the SV on the bottom side of $\mathrm{MoS}_{2}$, which is otherwise difficult to access. This unique property of MPS allows us to systematically compare three types of $\mathrm{MoS}_{2}$ samples: as-exfoliated on $\mathrm{SiO}_{2}$, top-side (TS) treated on $\mathrm{SiO}_{2}$ and double-side (DS) treated. For the MPS treatment, we used a liquid-phase process ${ }^{22}$, followed by $350^{\circ} \mathrm{C}$ annealing in forming gas to repair the SV (see Methods and Supplementary Fig. 3 for details of sample preparation and characterization).

We first study the reaction kinetics of SV and MPS by density functional theory (Fig. 1). The reaction can be described by $\mathrm{HS}\left(\mathrm{CH}_{2}\right)_{3} \mathrm{Si}\left(\mathrm{OCH}_{3}\right)_{3}+\mathrm{SV} \rightarrow \mathrm{CH}_{3}\left(\mathrm{CH}_{2}\right)_{2} \mathrm{Si}\left(\mathrm{OCH}_{3}\right)_{3}$ (Supplementary Fig. 4; Supplementary Movie 1), which is exothermic with the enthalpy change of $-333.3 \mathrm{~kJ} \mathrm{~mol}^{-1}$ of MPS. The reaction process comprises two steps with an energy barrier of $0.51 \mathrm{eV}$ and $0.22 \mathrm{eV}$, respectively. In the first step (Supplementary Fig. 5), MPS chemically absorbs onto an SV through the sulfur atom, and then it cleaves the S-H bond and forms a thiolate surface intermediate. The dissociated $\mathrm{H}$ atom is bonded to a neighbouring $S$ atom. The second step involves cleavage of the S-C bond and hydrogenation of the thiolate intermediate to form the final product trimethoxy(propyl)silane (Supplementary Fig. 6). We note that the two-step process agrees well with earlier thiol absorption experiments on defective $\mathrm{MoS}_{2}$ (refs 20,22). The relatively low energy barriers also facilitate the reaction to occur at low temperature.

To quantify the effect of MPS treatment on sample quality, we perform aberration-corrected transmission electron microscopy (TEM) on as-exfoliated and TS-treated monolayer $\mathrm{MoS}_{2}$. Figure 2 compares high-resolution TEM images of a typical as-exfoliated 


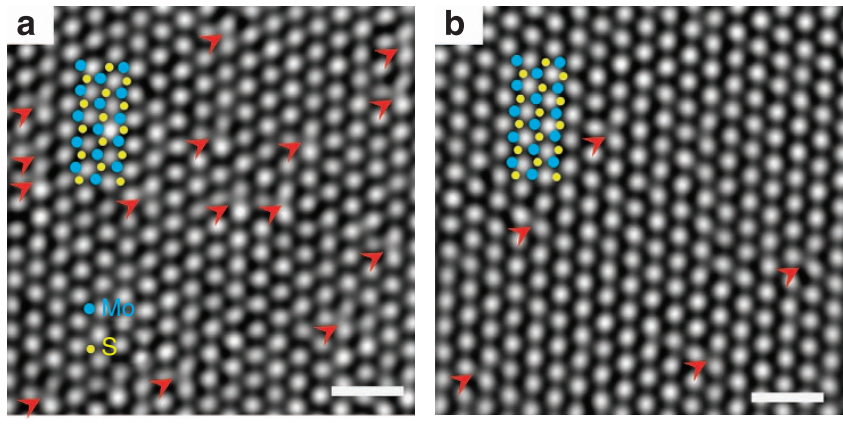

Figure 2 | High-resolution aberration-corrected TEM images.

(a) As-exfoliated and (b) TS-treated monolayer $\mathrm{MoS}_{2}$ sample, showing the significant reduction of SV by MPS treatment. The SVs are highlighted by red arrows. The overlaid blue and yellow symbols mark the position of Mo and $S$ atoms, respectively. Scale bar, $1 \mathrm{~nm}$. Detailed intensity profile analysis and histogram of SV density are shown in Supplementary Fig. 9.

and TS-treated sample. The SVs can be clearly distinguished by analysing the intensity profile ${ }^{11}$ as shown in Supplementary Fig. 9. Statistical analysis (from more than 15 areas in each case) indicates that the density of $\mathrm{SV}$ is reduced from $\sim 6.5 \times 10^{13} \mathrm{~cm}^{-2}$ for the as-exfoliated samples to $\sim 1.6 \times 10^{13} \mathrm{~cm}^{-2}$ for the TS-treated samples (Supplementary Fig. $9 c$,d). Owing to the difficulty in making TEM samples, we are not able to characterize the DS-treated $\mathrm{MoS}_{2}$, where further reduction of SV is expected. During the course of TEM experiment, we have paid great attention to minimize the knock-on damage and lattice reconstruction caused by electron beam irradiation ${ }^{24}$ by limiting the exposure time to $<30$ s and the current density to below $10^{6} \mathrm{enm}^{-2} \mathrm{~S}^{-1}$ (ref. 11). The SV generation rate induced by electron irradiation under our experimental conditions was very low, $\sim 5.6 \times 10^{10} \mathrm{~cm}^{-2} \mathrm{~S}^{-1}$ (ref. 11). Therefore, the SVs in Fig. 2 are believed to be intrinsic rather than induced by electron irradiation.

Electrical transport properties. Next, we systematically investigated the effect of MPS treatment on electrical transport properties of $\mathrm{MoS}_{2}$. We fabricated backgated field-effect transistors (FETs) on as-exfoliated, TS-treated and DS-treated monolayer $\mathrm{MoS}_{2}$ samples, and carried out electrical measurements in high vacuum with a standard four-probe technique, unless otherwise stated (see Methods for details of device fabrication and measurement). All the devices exhibited very small hysteresis at room temperature, which became even smaller as they were cooled down (Supplementary Fig. 10). Therefore, in the following we only present electrical data from the forward sweep.

Figure 3a shows the four-probe conductivity $\sigma=\left(I_{d s}\right) /(\Delta V)$ $(L) /(W)$ as a function of backgate voltage $V_{\mathrm{g}}$ for three representative devices at room temperature $(300 \mathrm{~K})$, where $I_{\mathrm{ds}}$ is the source-drain current; $\Delta V, L$ and $W$ are the voltage difference, distance and sample width between the two voltage probes, respectively. The MPS-treated samples show improved $\sigma$ compared with the as-exfoliated one, indicating higher sample and interface quality. At carrier density $n=C_{\mathrm{g}} V_{\mathrm{g}}=7.1 \times 10^{12}$ $\mathrm{cm}^{-2}\left(C_{\mathrm{g}}=11.6 \mathrm{nF} \mathrm{cm}^{-2}\right.$ is the gate capacitance for $300 \mathrm{~nm}$ $\mathrm{SiO}_{2}$ dielectrics), the DS-treated sample shows $\sigma=1.52 e^{2} h^{-1}$ and field-effect mobility $\mu=(\mathrm{d} \sigma) /\left(C_{\mathrm{g}} \mathrm{d} V_{\mathrm{g}}\right)=81 \mathrm{~cm}^{2} \mathrm{~V}^{-1} \mathrm{~s}^{-1}$. To our best knowledge, this is the highest room-temperature field-effect mobility reported so far for monolayer $\mathrm{MoS}_{2}$ regardless of the device geometry ${ }^{2,8-10}$.

To gain further insights into the charge transport physics, we performed variable-temperature electrical measurements down to $10 \mathrm{~K}$. Surprisingly, the three types of devices exhibit very different behaviour (Fig. 3; Supplementary Fig. 11). For the as-exfoliated sample, $\sigma$ monotonically decreases during cooling over the entire range of $n$, indicating an insulating behaviour (Fig. 3d; Supplementary Fig. 11a). For the DS-treated sample, the transfer curves all intersect near $V_{\mathrm{g}}=80 \mathrm{~V}$ (corresponding to $n \sim 5.7 \times 10^{12} \mathrm{~cm}^{-2}$, Supplementary Fig. 11c), which is a signature of MIT. Metallic and insulating behaviours are observed down to the base temperature for $n>6.6 \times 10^{12} \mathrm{~cm}^{-2}$ and $n<3.5 \times 10^{12} \mathrm{~cm}^{-2}$, respectively. At intermediate $n$, the $\sigma-T$ characteristics are not monotonic, and MIT occurs within our experimental temperature range (solid symbols in Fig. 3f). For the TS-treated sample, MIT is observed for $n>4.7 \times 10^{12} \mathrm{~cm}^{-2}$ (solid symbols in Fig. 3e), while insulating behaviour is always observed at low temperatures (Fig. 3e; Supplementary Fig. 11b). As we shall see later, these distinct transport behaviours precisely reflect the quality of the $\mathrm{MoS}_{2}$ and interface.

The scaling behaviour of $\mu$ is also very different for the three samples (Fig. 3b,c). The as-exfoliated sample shows the lowest $\mu$ among the three samples. The $\mu-T$ curve is not monotonic with a peak value of $31 \mathrm{~cm}^{2} \mathrm{~V}^{-1} \mathrm{~s}^{-1}$ near $175 \mathrm{~K}$. In contrast, $\mu$ monotonically increases for the MPS-treated samples on cooling. For $T>100 \mathrm{~K}, \mu \sim T^{-\gamma}$, where $\gamma=0.72$ and 0.64 for the DS-treated and TS-treated samples, respectively. For $T<100$ $\mathrm{K}, \mu$ gradually saturates. At $T=10 \mathrm{~K}, \mu=320 \mathrm{~cm}^{2} \mathrm{~V}^{-1} \mathrm{~s}^{-1}$ for the DS-treated sample, which is $\sim 3$ (22) times higher than the TS-treated (as-exfoliated) one.

We stress that all the above-mentioned transport phenomena are reproducible among different samples. In Supplementary Fig. 12, we show three additional sets of data for two-terminal devices. Although the mobility is lower than their corresponding four-terminal counterparts due to contact resistance, all the key transport properties, including MIT, scaling of mobility and conductivity, are qualitatively reproduced.

Theoretical modelling of charge transport. Although some theories have been proposed to explain the mobility of monolayer $\mathrm{MoS}_{2}$ (refs 13,25), a more complete physical model remains to be developed to fully understand the charge transport. In particular, the model should establish the correlation between different transport regimes and the underlying microscopic scattering mechanisms, provide quantitative information about the samples, and project device performance based on realistic parameters.

We start by calculating the mobility of monolayer $\mathrm{MoS}_{2}$. According to Matthiessen's rule, the mobility for free carriers is expressed as

$$
\mu_{0}(n, T)^{-1}=\mu_{\mathrm{ph}}(T)^{-1}+\mu_{\mathrm{CI}}(n, T)^{-1}+\mu_{\mathrm{sr}}^{-1}
$$

where $\mu_{\mathrm{ph}}, \mu_{\mathrm{CI}}$ and $\mu_{\mathrm{sr}}$ are mobility limited by phonons, CI and short-range scatterings, respectively (the calculation of each term is described in Methods). The incorporation of $\mu_{\mathrm{sr}}$ in our model is motivated by (i) TEM characterization that clearly shows the existence of short-range SV defects and (ii) the saturation of $\mu$ at low temperatures. We do not consider surface optical phonon scattering because the relatively high energy of the $\mathrm{SiO}_{2}$ phonon modes makes them irrelevant to the low-field mobility phenomenon considered here ${ }^{25}$.

The experimentally measured 'effective' field-effect mobility $\mu$ is not equal to the free-carrier mobility $\mu_{0}$, due to the presence of charge traps that limits the free carrier population. Recently, localized trap states within the bandgap have been observed in both exfoliated and CVD monolayer $\mathrm{MoS}_{2}$ (refs 11,14,15). The impurity band from these trap states can introduce a mobility edge that strongly affects the charge transport ${ }^{26}$. In a simple picture $^{27}$, transport is carried only by electrons in the extended states, that is, states above the mobility edge. This model, which 
a

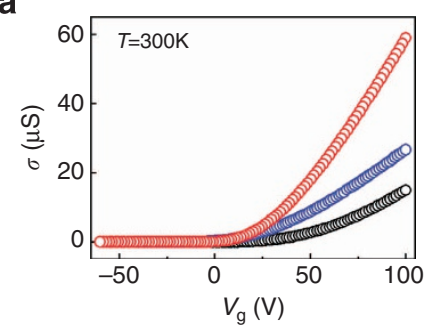

d

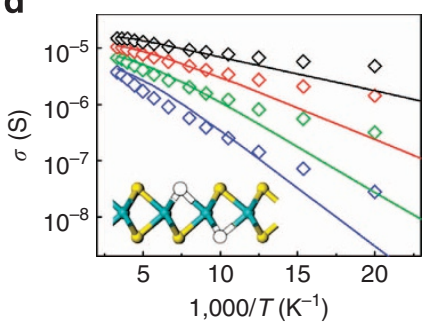

b

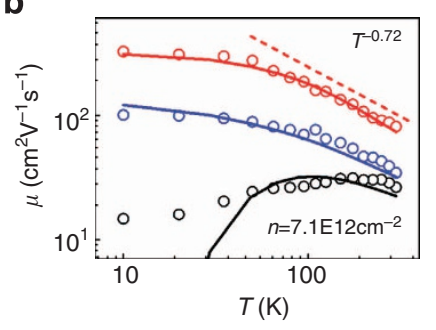

e

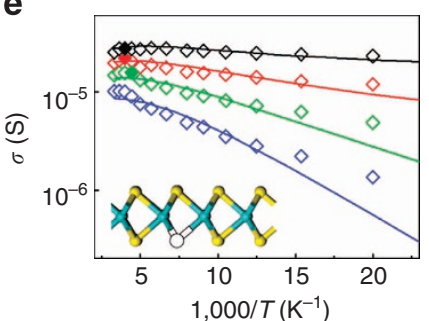

C

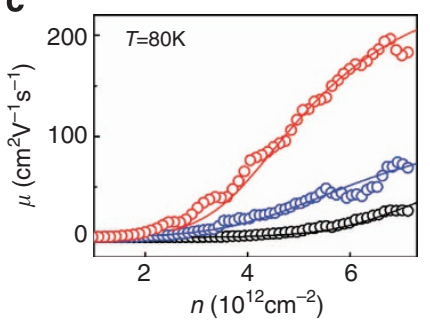

f

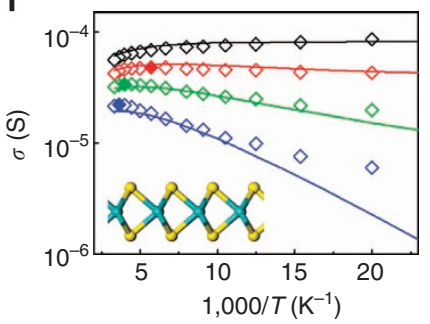

Figure 3 | The effect of defect and interface engineering on monolayer $\mathbf{M o S}_{\mathbf{2}}$ charge transport. (a) Typical $\sigma$ - $V_{\mathrm{g}}$ characteristics for as-exfoliated (black), TS-treated (blue) and DS-treated (red) monolayer $\mathrm{MoS}_{2}$ at $T=300 \mathrm{~K}$. (b) $\mu$-T characteristics for the three devices at $n=7.1 \times 10^{12} \mathrm{~cm}-2$. Solid lines are the best theoretical fittings. The dashed red line shows $T^{-0.72}$ scaling. (c) $\mu$-n characteristics for the three devices at $T=80 \mathrm{~K}$. Solid lines are the best theoretical fittings. (d-f) Arrhenius plot of $\sigma$ (symbols) and theoretical fittings (lines) for the as-exfoliated (d), TS-treated (e) and DS-treated (f) $\mathrm{MoS}_{2}$. From top to bottom, $n=7.0,6.0,5.0$ and $4.0 \times 10^{12} \mathrm{~cm}^{-2}$, respectively. The critical points of the MIT are highlighted by solid symbols in (e) and (f). Insets in (d-f) show the cartoon illustration of the corresponding $\mathrm{MoS}_{2}$ samples undergone different treatments.

Table 1 | The fitting parameters in our theoretical model for the three devices in Fig. 3.

\begin{tabular}{|lccc} 
& As-exfoliated & TS-treated & DS-treated \\
\hline$N_{\mathrm{i}}\left(10^{12} \mathrm{~cm}^{-2}\right)$ & 0.7 & 0.59 & 0.24 \\
$N_{\mathrm{tr}}\left(10^{12} \mathrm{~cm}^{-2}\right)$ & 8.1 & 6.16 & 5.22 \\
$\Delta E_{\mathrm{tr}}\left(\mathrm{meV}^{2}\right)$ & 75 & 46 & 58 \\
$\mu_{\mathrm{sr}}\left(\mathrm{cm}^{2} \mathrm{~V}^{-1} \mathrm{~s}^{-1}\right)$ & 127 & 161 & 410 \\
\hline
\end{tabular}

does not account for the hopping between the localized states, has been very successful in modelling organic $\mathrm{FETs}^{27,28}$. Here we adopt the same model to extract important physical quantities such as the density of $\mathrm{CI}\left(N_{\mathrm{i}}\right)$ and trap states $\left(N_{\mathrm{tr}}\right)$, while avoiding the complexity of dealing with the energy-dependent mobility and percolation effects in hopping transport ${ }^{27}$ (the detailed calculation is described in Methods). The model is further justified by its excellent agreement with the experimental data over a broad range of temperature and carrier density.

\section{Discussion}

With the above model, we can now quantitatively understand the obtained experimental data. The solid lines in Fig. 3 are the best fitting results using the parameters listed in Table 1. Remarkably, the scaling of mobility and conductivity with temperature and carrier density is well reproduced with a single set of parameters, suggesting that our model captures the essential physics. At low temperature and low carrier density, the calculated $\sigma$ and $\mu$ are lower than experiments (Fig. 3b,d-f), presumably due to the omission of hopping transport in our model. Hence, the discrepancy is the largest for the as-exfoliated sample (Fig. 3b,d) as $N_{\text {tr }}$ is the highest.

The fitting parameters in Table 1 give considerable insights into the microscopic origin of impurities in $\mathrm{MoS}_{2}$. In all of our samples, $\mu$ is much lower than $\mu_{\mathrm{ph}}$, indicating that phonon scattering does not play a significant role. Rather, the mobility is largely limited by $\mathrm{CI}$ and short-range scatterings. We notice that $N_{\mathrm{i}}$ and $\mu_{\mathrm{sr}}$ are reduced by MPS treatment and are correlated for each sample, suggesting that CI partially shares the microscopic origin with short-range defects, most likely SV. CI also partially comes from the interface, as the DS-treated sample has much lower $N_{\mathrm{i}}$ than TS-treated one. For the DS-treated sample, $N_{\mathrm{i}}$ becomes comparable to that of $\mathrm{SiO}_{2}$ substrate $(0.24-2.7 \times$ $10^{11} \mathrm{~cm}^{-2}$ ) (refs 29-31), indicating that a large portion of SV is repaired. $N_{\mathrm{tr}}$ is also partially due to SVs as it can be reduced by MPS treatment. However, $N_{\mathrm{tr}}$ is an order of magnitude higher than $N_{\mathrm{i}}$ for all the samples, pointing to contributions from additional sources. This could be due to the ambient species absorbed between $\mathrm{MoS}_{2}$ and $\mathrm{SiO}_{2}$ during exfoliation, which act as charge traps as in the case of graphene ${ }^{29,31,32}$.

For the MPS-treated samples, the high-temperature mobility is limited by $\mathrm{CI}$, leading to the usual $T^{-\gamma}$ scaling behaviour. Reference 13 predicts $\gamma \sim 1$ for the range of carrier densities studied here. However, both short-range scattering (temperature independent) and thermal excitation from charge traps (which leads to the opposite trend since the density of electrons in the extended states $n_{\mathrm{c}}$ increases with temperature) can decrease the effective $\gamma$ as observed here. Therefore, one cannot reliably infer the scattering mechanism solely by analysing $\gamma$. When the charge traps become dominant (which usually happens at low temperature and low carrier density), the mobility even exhibits an insulating behaviour as commonly observed in backgated devices ${ }^{2,11,12}$ and in the as-exfoliated sample here (Fig. 3b).

The mobility increases with carrier density for all three samples from the combined effect of CI and charge traps (Fig. 3c). Below a threshold carrier density (equivalent to mobility edge), $\mu$ becomes negligible due to the localized nature of electrons in charge traps. The mobility edge is found to decrease with temperature (Supplementary Fig. 13) because of the thermal excitation of electrons to the extended states. At $T=80 \mathrm{~K}$, the threshold density is about $2 \times 10^{12}, 3 \times 10^{12}$ and $5 \times 10^{12} \mathrm{~cm}^{-2}$ for the DS-treated, TS-treated and as-exfoliated samples, respectively (Fig. 3c). These values are on the same order of magnitude with $N_{\text {tr }}$, further supporting the charge trap model. 
a

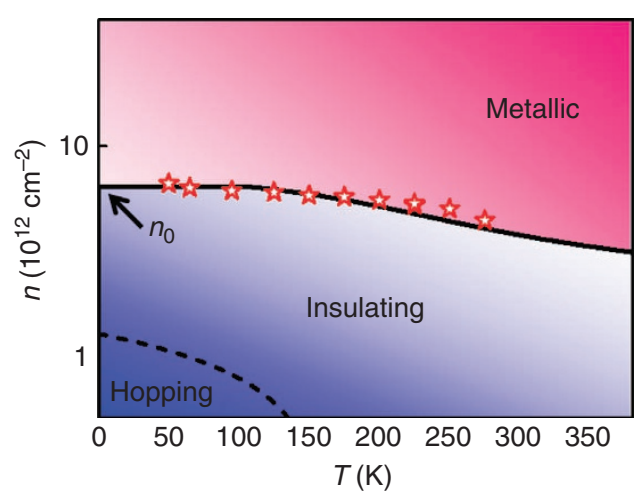

b

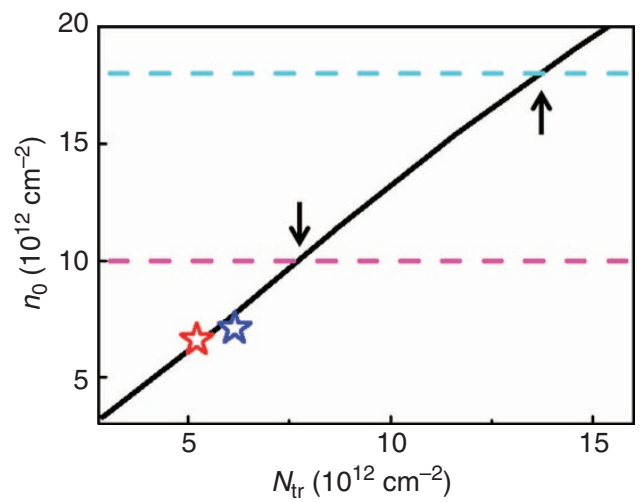

c

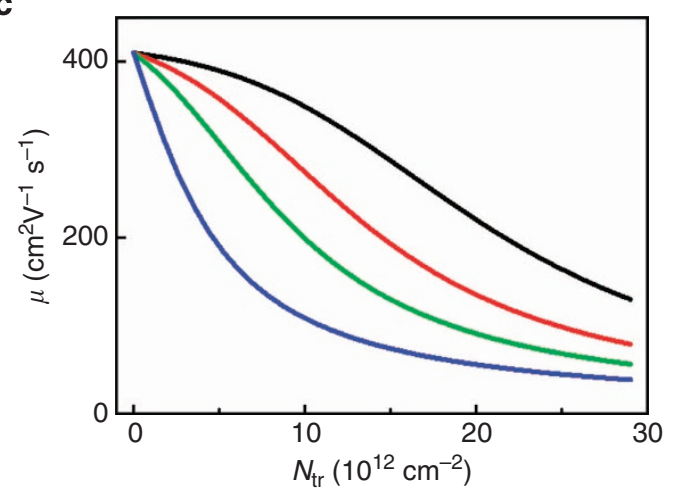

Figure 4 | Theory of charge transport in $\mathbf{M o S}_{2}$. (a) Phase diagram of charge transport in monolayer $\mathrm{MoS}_{2}$. The solid black line plots the calculated MIT critical points (using the parameters of DS-treated sample in Table 1) that separate the metallic and insulating regimes. The red symbols are experimental MIT points extracted from Fig. 3f. The lower left corner of the phase diagram illustrates the hopping transport regime (not calculated). (b) The solid black line is the calculated $n_{0}$ as a function of $N_{\text {tr }}$ using the parameters of DS-treated sample in Table 1. The MIT curves under different $N_{t r}$ are plotted in Supplementary Fig. 14. The blue and red symbols are experimental points from the TS-treated and DS-treated samples in Fig. 3, respectively. We use the highest $n$ that exhibits MIT as $n_{0}$. The horizontal dashed lines are MIT critical density estimated from refs 2,9, respectively. The intersections with the solid line represent the estimate of $N_{\mathrm{tr}}$ in their devices (black arrows). (c) Theoretical calculation of $\mu$ as a function of $N_{\text {tr }}$ at $T=300 \mathrm{~K}$ without any $\mathrm{Cl}$ or short-range scatterings (using the parameters of DS-treated sample in Table 1). From top to bottom, $n=2.0$, $1.2,0.7$ and $0.16 \times 10^{13} \mathrm{~cm}^{-2}$, respectively. The phonon-limited value of $410 \mathrm{~cm}^{2} \mathrm{~V}^{-1} \mathrm{~s}^{-1}$ is recovered at $N_{\mathrm{tr}}=0$.

Finally, we can also understand MIT in the framework of charge traps. Strong electron-electron correlation in 2D electron gas has been proposed to explain the MIT in monolayer $\mathrm{MoS}_{2}$, which gives a universal threshold density $n_{\mathrm{MIT}} \sim 10^{13} \mathrm{~cm}^{-2}$ (ref. 2). In our experiments, however, $n_{\text {MIT }}$ is apparently dependent on sample quality (Fig. 3). The MIT can be intuitively understood from equation (6) (Methods). When $n<N_{\text {tr }}$, the density of conducting electrons in the extended states $\left(n_{c}\right)$ is exponentially dependent on temperature due to thermal activation, the temperature dependence of $\sigma$ is dominated by $n_{c}$, showing thermally activated insulating behaviour ${ }^{2}, 11,12,14$. For even smaller $n$ and $T$, transport is dominated by variable-range hopping because $n_{c}$ can be ignored ${ }^{11,14}$ (Fig. 4a). When $n>N_{t r}$, the Fermi level is above the mobility edge and $n_{\mathrm{c}}$ is independent of temperature. Thus, the temperature dependence of $\sigma$ is dominated by $\mu_{0}$, showing metallic behaviour. Therefore, MIT occurs when $n \approx N_{\text {tr }}$. Using the trap and CI parameters of the DStreated sample in Table 1 , we numerically calculated $\sigma(n, T)$ (equation (6)) and obtained the $n$ and $T$ of each critical point as in Fig. 3f. Figure 4a shows the calculated transport phase diagram with metallic and insulating regions. Excellent agreement with experiment is achieved without any fitting parameters, showing the consistency of our model. From Fig. $4 \mathrm{a}, n_{\mathrm{MIT}}$ slowly increases with decreasing temperature and converges to $n_{0}$ at $T=0 \mathrm{~K}$. When $n>n_{0}$, metallic behaviour is always expected. When $n<n_{0}$, MIT is always expected at finite temperature. Under realistic trap and CI parameters, $n_{0}$ is linearly proportional to, but slightly higher than $N_{\text {tr }}$ (Fig. 4b; Supplementary Fig. 14). Therefore, $n_{0}$ can be used as a rough estimate of $N_{\mathrm{tr}}$. After careful comparison with literature $2,9,14,33$, we find that the $N_{\text {tr }}$ of our MPS-treated samples is indeed the lowest (Fig. 4b), indicating the highest sample quality.

From the above discussion, it is clear that the transport in current state-of-the-art monolayer $\mathrm{MoS}_{2}$ samples is still limited by charge traps, CI and short-range defects. To reach the real potential of monolayer $\mathrm{MoS}_{2}$ in high-performance devices, continuous improvement of sample and interface quality is still needed. In Fig. 4c, we project the room-temperature mobility as a function of $N_{\mathrm{tr}}$ in the ideal case, that is, without CI and shortrange scattering. The mobility at low carrier density is rapidly degraded by even a small $N_{\mathrm{tr}}$. At a modest $n=7 \times 10^{12} \mathrm{~cm}^{-2}, N_{\mathrm{tr}}$ has to be lower than $8.8 \times 10^{11} \mathrm{~cm}^{-2}$, which is $\sim 6$ times lower than our DS-treated device, to achieve mobility $>400 \mathrm{~cm}^{2} \mathrm{~V}^{-1} \mathrm{~s}^{-1}$.

In conclusion, we have shown that thiol chemistry is an effective approach to engineer the defects and interface in monolayer $\mathrm{MoS}_{2}$ towards intrinsic charge transport. A physical model that includes charge traps and major scattering sources has been developed to comprehensively describe the charge transport in $\mathrm{MoS}_{2}$ and to quantify the density of CI and charge traps in the samples. We believe that our model captures the essential charge transport physics for monolayer $\mathrm{MoS}_{2}$ and can be readily extended to other $2 \mathrm{D}$ semiconductors ${ }^{33-35}$.

\section{Methods}

Density functional theory calculations. Density functional theory calculations were performed using the Vienna $a b$ initio simulation package (VASP) ${ }^{36,37}$. Projector-augmented-wave $\mathrm{e}^{38}$ potentials were used to describe ion-electron interactions, and the exchange correlation potential was represented by the local density approximation ${ }^{39}$. We used the climbing-image nudged elastic band method to locate the minimum energy paths and the transition states ${ }^{40}$. The defective $\mathrm{MoS}_{2}$ sheet was modelled by a $4 \times 4$ supercell of $\mathrm{MoS}_{2}$ with a single SV. A k-point sampling of $5 \times 5 \times 1$ was used for all the calculations.

$\mathbf{M o S}_{2}$ sample preparation and MPS treatment. In this work, we exfoliated monolayer $\mathrm{MoS}_{2}$ from natural bulk flakes (SPI Supplies). The as-exfoliated samples were directly exfoliated on $300 \mathrm{~nm} \mathrm{SiO} 2 / \mathrm{Si}$ substrate. Before device fabrication, the samples were annealed in a mixture of $\mathrm{H}_{2} / \mathrm{Ar}$ at $350{ }^{\circ} \mathrm{C}$ to remove organic residue.

For the TS-treated samples, we first exfoliated monolayer $\mathrm{MoS}_{2}$ on $300 \mathrm{~nm}$ $\mathrm{SiO}_{2} / \mathrm{Si}$ substrate, followed by annealing in a mixture of $\mathrm{H}_{2} / \mathrm{Ar}$ at $350{ }^{\circ} \mathrm{C}$ to remove organic residue. The sample was then dipped in a fresh solution of $1 / 15(\mathrm{v} / \mathrm{v})$ 
MPS/dichloromethane for $24 \mathrm{~h}$ in a dry glove box to grow MPS on $\mathrm{MoS}_{2}$. After growth, the sample was taken out, rinsed thoroughly with dichloromethane and isopropanol (IPA), and blown dry with $\mathrm{N}_{2}$. Finally, the sample was annealed in a mixture of $\mathrm{H}_{2} / \mathrm{Ar}$ at $350^{\circ} \mathrm{C}$ for $20 \mathrm{~min}$ to finish the MPS treatment and remove the extra MPS on $\mathrm{MoS}_{2}$ (Supplementary Fig. 3).

For DS-treated samples, the $300 \mathrm{~nm} \mathrm{SiO}_{2} / \mathrm{Si}$ substrate was first subject to a 30-min UV/ozone treatment to enhance surface hydrophilicity. The substrate was then dipped in a $10 \%(\mathrm{v} / \mathrm{v})$ MPS/dichloromethane solution for $12 \mathrm{~h}$ in a dry glove box to grow MPS SAM. After the SAM growth, the substrate was sonicated in dichloromethane followed by thorough rinsing with dichloromethane and IPA, and drying with $\mathrm{N}_{2}$ (Supplementary Fig. 3). We then exfoliated monolayer $\mathrm{MoS}_{2}$ on the SAM-treated $\mathrm{SiO}_{2} / \mathrm{Si}$ substrate. After exfoliation, the sample was dipped in a fresh solution of $1 / 15(\mathrm{v} / \mathrm{v}) \mathrm{MPS} /$ dichloromethane for $24 \mathrm{~h}$ in a dry glove box to grow MPS on the top side of $\mathrm{MoS}_{2}$, followed by thorough rinsing. Finally, the sample was annealed in a mixture of $\mathrm{H}_{2} / \mathrm{Ar}$ at $350^{\circ} \mathrm{C}$ for $20 \mathrm{~min}$ to finish the MPS treatment.

Device fabrication and electrical measurement. We used standard photolithography to pattern the electrodes of $\mathrm{MoS}_{2}$ backgated FETs on $300 \mathrm{~nm} \mathrm{SiO} / 2 \mathrm{Si}$ substrates. Ti/Pd $(20 \mathrm{~nm} / 20 \mathrm{~nm})$ was electron beam evaporated as the contact metal for source, drain and voltage probes, followed by lift-off. All devices were annealed at $350^{\circ} \mathrm{C}$ in Ar to improve contacts. Electrical measurements were carried out by an Agilent B1500 semiconductor parameter analyser in a close-cycle cryogenic probe station with base pressure $\sim 10^{-5}$ Torr. Before electrical measurements, a vacuum annealing at base pressure was performed to remove adsorbates and improve device performances ${ }^{41}$.

TEM characterizations. To prepare TEM samples, monolayer $\mathrm{MoS}_{2}$ was first exfoliated on $300 \mathrm{~nm} \mathrm{SiO}_{2} / \mathrm{Si}$ substrate. We then align-transferred the sample to a copper TEM grid by the PMMA-based transfer method described in ref. 11 .

The high-resolution TEM images were recorded in an image aberrationcorrected TEM (FEI Titan 80-300 at $80 \mathrm{kV}$ ) equipped with a charge-coupled device camera (GatanUltraScanTM 1000). The exposure time was $1 \mathrm{~s}$. Before recording, we set the third-order spherical aberration in the range of $1-6 \mu \mathrm{m}$ and then we recorded the images under slightly underfocused. To minimize irradiation damage and prevent new SVs generating during normal imaging ${ }^{24}$, we limited the total exposure time to $<30 \mathrm{~s}$ and the current density to $<10^{6} \mathrm{enm}^{-2} \mathrm{~s}^{-1}$. A band-pass filter to the Fast Fourier Transformation of the original images was applied to enhance the visibility of the defects.

Theoretical modelling. The phonon-limited mobility is numerically calculated according to ref. 7:

$$
\mu_{\mathrm{ph}}(T)=\left\{\begin{array}{c}
\alpha_{1} T^{-1}, T<100 \mathrm{~K} \\
\alpha_{2} T^{-1.69}, T \geq 100 \mathrm{~K}
\end{array}\right.
$$

where the coefficients $\alpha_{1}=2.625 \times 10^{5} \mathrm{~cm}^{2} \mathrm{KV}^{-1} \mathrm{~s}^{-1}$ and $\alpha_{2}=6.297$ $\times 10^{6} \mathrm{~cm}^{2} \mathrm{KV}^{-1} \mathrm{~s}^{-1}$ satisfy the boundary condition that $\mu_{\mathrm{ph}}(300 \mathrm{~K})$ $=410 \mathrm{~cm}^{2} \mathrm{~V}^{-1} \mathrm{~s}^{-1}$ and that $\mu_{\mathrm{ph}}(T)$ is continuous at $100 \mathrm{~K}$. The transition at $100 \mathrm{~K}$ is due to the different phonon modes that limit the mobility ${ }^{7}$.

The CI-limited mobility is calculated by the model described in ref. 13:

$$
\mu_{\mathrm{CI}}(n, T)=\frac{e}{\pi n \hbar^{2} k_{\mathrm{B}} T} \int_{0}^{\infty} f(E)[1-f(E)] \Gamma_{\mathrm{imp}}(E)^{-1} E d E
$$

where $f(E)$ is the equilibrium Fermi-Dirac distribution function and $\Gamma_{\text {imp }}$ is the electron momentum relaxation rate given by

$$
\Gamma_{\text {imp }}=\frac{N_{\mathrm{i}}}{2 \pi \hbar} \int d k^{\prime}\left|\varphi_{\left|k-k^{\prime}\right|}^{s c r}\right|^{2}\left(1-\cos \theta_{k k^{\prime}}\right) \delta\left(E_{k}-E_{k^{\prime}}\right)
$$

where $\phi_{|q|}^{\mathrm{scr}}$ is the screened scattering potential for a single CI. The expression and derivation for $\phi_{|q|}^{\text {scr }}$ can be found in ref. 13. Physically speaking, $\phi_{|q|}^{\text {scr }}$ includes the effect of temperature-dependent charge polarizability on the electron screening of the charged impurity, which dominates the temperature dependence of the mobility at low and room temperature. The expression also implies that $\mu_{\mathrm{CI}}$ is inversely proportional to $N_{\mathrm{i}}$. Hence, from the fitting of the $\mu_{\mathrm{CI}}$ to the total effective mobility data, we are able to quantify the $N_{\mathrm{i}}$ within the $\mathrm{MoS}_{2}$. As an example, Supplementary Fig. 15 plots $\mu_{\mathrm{CI}}$ as a function of $T$ under $N_{\mathrm{i}}=10^{12} \mathrm{~cm}^{-2}$.

The short-range scattering-limited mobility $\mu_{\text {sr }}$ is a constant fitting parameter that does not depend on temperature or carrier density ${ }^{8}$.

To model the effect of charge traps, we assume the charge traps are uniformly distributed within $\Delta E_{\mathrm{tr}}$ below the conduction band edge (Supplementary Fig. 16). We only consider shallow traps as the density of deep traps is at least an order of magnitude lower ${ }^{14}$, and that deep traps do not affect mobility and conductivity ${ }^{27}$. The Fermi energy $E_{\mathrm{F}}(n, T)$ is determined by

$$
n=C_{\mathrm{g}} V_{\mathrm{g}}=\int_{0}^{+\infty} N_{0} \frac{1}{e^{\left(E-E_{\mathrm{F}}\right) / k_{\mathrm{B}} T}+1} d E+\int_{-\Delta E_{\mathrm{tr}}}^{0} \frac{N_{\mathrm{tr}}}{\Delta E_{\mathrm{tr}}} \frac{1}{e^{\left(E-E_{\mathrm{F}}\right) / k_{\mathrm{B}} T}+1} d E
$$

where $N_{0}=\left(2 m^{*}\right) /\left(\pi \hbar^{2}\right)=3.8 \times 10^{14} \mathrm{eV}^{-1} \mathrm{~cm}^{-2}$ is the density of states in the conduction band, and $m^{*}=0.45 m_{e}$ is the conduction band effective mass ${ }^{42}$. The density of conducting electrons in the extended states is

$$
n_{\mathrm{c}}(n, T)=\int_{0}^{+\infty} N_{0} \frac{1}{e^{\left(E-E_{\mathrm{F}}\right) / k_{\mathrm{B}} T}+1} d E
$$

The conductivity is calculated by

$$
\sigma(n, T)=e n_{\mathrm{c}}(n, T) \mu_{0}(n, T)
$$

and the 'effective' mobility is given by

$$
\mu(n, T)=\mu_{0}(n, T) \frac{\partial n_{\mathrm{c}}(n, T)}{\partial n}
$$

\section{References}

1. Liu, L., Kumar, S. B., Ouyang, Y. \& Guo, J. Performance limits of monolayer transition metal dichalcogenide transistors. IEEE Trans. Electron Devices $\mathbf{5 8}$, 3042-3047 (2011).

2. Radisavljevic, B. \& Kis, A. Mobility engineering and a metal-insulator transition in monolayer $\mathrm{MoS}_{2}$. Nat. Mater. 12, 815-820 (2013).

3. Wang, H. et al. Integrated circuits based on bilayer MoS2 transistors. Nano Lett. 12, 4674-4680 (2012).

4. Chuang, S. et al. $\mathrm{MoS}_{2}$ P-type transistors and diodes enabled by high work function $\mathrm{MoO}_{\mathrm{x}}$ contacts. Nano Lett. 14, 1337-1342 (2014).

5. Yu, W. et al. Highly efficient gate-tunable photocurrent generation in vertical heterostructures of layered materials. Nat. Nanotech. 8, 952-958 (2013).

6. Lopez-Sanchez, O., Lembke, D., Kayci, M., Radenovic, A. \& Kis, A. Ultrasensitive photodetectors based on monolayer $\mathrm{MoS}_{2}$. Nat. Nanotech. 8, 497-501 (2013).

7. Kaasbjerg, K., Thygesen, K. S. \& Jacobsen, K. W. Phonon-limited mobility in n-type single-layer $\mathrm{MoS}_{2}$ from first principles. Phys. Rev. B 85, 115317 (2012)

8. Schmidt, H. et al. Transport properties of monolayer $\mathrm{MoS}_{2}$ grown by chemical vapour deposition. Nano Lett. 14, 1909-1913 (2014).

9. Baugher, B. W., Churchill, H. O., Yang, Y. \& Jarillo-Herrero, P. Intrinsic electronic transport properties of high-quality monolayer and bilayer $\mathrm{MoS}_{2}$ Nano Lett. 13, 4212-4216 (2013).

10. Liu, H. et al. Statistical study of deep submicron dual-gated field-effect transistors on monolayer chemical vapor deposition molybdenum disulfide films. Nano Lett. 13, 2640-2646 (2013).

11. Qiu, H. et al. Hopping transport through defect-induced localized states in molybdenum disulphide. Nat. Commun. 4, 2642 (2013).

12. Ghatak, S., Pal, A. N. \& Ghosh, A. Nature of electronic states in atomically thin $\mathrm{MoS}_{2}$ field-effect transistors. ACS Nano 5, 7707-7712 (2011).

13. Ong, Z. Y. \& Fischetti, M. V. Mobility enhancement and temperature dependence in top-gated single-layer $\mathrm{MoS}_{2}$. Phys. Rev. B 88, 165316 (2013).

14. Zhu, W. et al. Electronic transport and device prospects of monolayer molybdenum disulphide grown by chemical vapour deposition. Nat. Commun. 5, 3087 (2014).

15. Ghatak, S., Mukherjee, S., Jain, M., Das Sarma, D. D. \& Ghosh, A. Microscopic origin of charged impurity scattering and flicker noise in $\mathrm{MoS}_{2}$ field-effect transistors, preprint at http://arxiv.org/abs/1403.3333 (2014).

16. Najmaei, S. et al. Tailoring the physical properties of molybdenum disulphide monolayers by control of interfacial chemistry. Nano Lett. 14, 1354-1361 (2014).

17. Chan, M. et al. Suppression of thermally activated carrier transport in atomically thin $\mathrm{MoS}_{2}$ on crystalline hexagonal boron nitride substrates. Nanoscale 5, 9572-9576 (2013).

18. Jin, T., Kang, J., Kim, E. S., Lee, S. \& Lee, C. Suspended single-layer $\mathrm{MoS}_{2}$ devices. J. Appl. Phys. 114, 164509 (2013).

19. Komsa, H. P., Kurasch, S., Lehtinen, O., Kaiser, U. \& Krasheninnikov, A. V. From point to extended defects in two-dimensional $\mathrm{MoS}_{2}$ : Evolution of atomic structure under electron irradiation. Phys. Rev. B 88, 035301 (2013).

20. Wiegenstein, C. G. \& Schulz, K. H. Methanethiol adsorption on defective $\mathrm{MoS}_{2}$ (0001) surfaces. J. Phys. Chem. B 103, 6913-6918 (1999).

21. Peterson, S. L. \& Schulz, K. H. Ethanethiol decomposition pathways on $\mathrm{MoS}_{2}$ (0001). Langmuir 12, 941-945 (1996).

22. Makarova, M., Okawa, Y. \& Aono, M. Selective adsorption of thiol molecules at sulfur vacancies on MoS2 (0001), followed by vacancy repair via S-C dissociation. J. Phys. Chem. C 116, 22411-22416 (2012).

23. Onclin, S., Ravoo, B. J. \& Reinhoudt, D. N. Engineering silicon oxide surfaces using self-assembled monolayers. Angew. Chem. Int. Ed. 44, 6282-6304 (2005)

24. Komsa, H. P. et al. Two-dimensional transition metal dichalcogenides under electron irradiation: defect production and doping. Phys. Rev. Lett. 109, 035503 (2012).

25. Ma, N. \& Jena, D. Charge scattering and mobility in atomically thin semiconductors. Phys. Rev. X 4, 011043 (2014). 
26. Ando, T., Fowler, A. B. \& Stern, F. Electronic properties of two-dimensional systems. Rev. Mod. Phys. 54, 437-672 (1982).

27. Xie, H., Alves, H. \& Morpurgo, A. F. Quantitative analysis of density-dependent transport in tetramethyltetraselenafulvalene single-crystal transistors: intrinsic properties and trapping. Phys. Rev. B 80, 245305 (2009).

28. Salleo, A. et al. Intrinsic hole mobility and trapping in a regioregular poly(thiophene). Phys. Rev. B 70, 115311 (2004).

29. Burson, K. et al. Direct imaging of charged impurity density in common graphene substrates. Nano Lett. 13, 3576-3580 (2013).

30. Deshpande, A., Bao, W., Miao, F., Lau, C. N. \& LeRoy, B. J. Spatially resolved spectroscopy of monolayer graphene on $\mathrm{SiO}_{2}$. Phys. Rev. B 79, 205411 (2009).

31. Martin, J. et al. Observation of electron-hole puddles in graphene using a scanning single-electron transistor. Nat. Phys. 4, 144-148 (2008).

32. Zhang, Y., Brar, V. W., Girit, C., Zettl, A. \& Crommie, M. F. Origin of spatial charge inhomogeneity in graphene. Nat. Phys. 5, 722-726 (2009).

33. Li, L. et al. Black phosphorus field-effect transistors. Nat. Nanotech. 9, 372-377 (2014).

34. Fang, H. et al. High-performance single layered WSe $\mathrm{W}_{2} \mathrm{p}-\mathrm{FET}$ s with chemically doped contacts. Nano Lett. 12, 3788-3792 (2012).

35. Larentis, S., Fallahazad, B. \& Tutuc, E. Field-effect transistors and intrinsic mobility in ultra-thin $\mathrm{MoSe}_{2}$ layers. Appl. Phys. Lett. 101, 223104 (2012).

36. Kresse, G. \& Hafner, J. Ab initio molecular dynamics for open-shell transition metals. Phys. Rev. B 48, 13115-13118 (1993).

37. Kresse, G. \& Furthmüller, J. Efficiency of $a b$-initio total energy calculations for metals and semiconductors using a plane-wave basis set. Comput. Mater. Sci. 6, 15-50 (1996).

38. Blöchl, P. E. Projector augmented-wave method. Phys. Rev. B 50, 17953-17979 (1994)

39. Ceperley, D. M. Ground state of the electron gas by a stochastic method. Phys. Rev. Lett. 45, 566-569 (1980).

40. Henkelman, G., Uberuaga, B. P. \& Jónsson, H. A climbing image nudged elastic band method for finding saddle points and minimum energy paths. J. Chem. Phys. 113, 9901 (2000).

41. Qiu, H. et al. Electrical characterization of back-gated bi-layer field-effect transistors and the effect of ambient on their performances. Appl. Phys. Lett. 100, 123104 (2012)
42. Yoon, Y., Ganapathi, K. \& Salahuddin, S. How good can monolayer $\mathrm{MoS}_{2}$ transistors be? Nano Lett. 11, 3768-3773 (2011).

\section{Acknowledgements}

This work was supported in part by Chinese National Key Fundamental Research Project 2013CBA01604, 2011CB922103, 2011CB707601, 2010CB923401, 2011CB302004; National Natural Science Foundation of China 61325020, 61261160499, 11274154 61229401, 21173040, 21373045, 113279028, 61274114; National Science and Technology Major Project 2011ZX02707; Natural Science Foundation of Jiangsu Province BK2012302, BK20130055, BK20130016, BK2012024; Specialized Research Fund for the Doctoral Program of Higher Education 20120091110028, and a project funded by the Priority Academic Program Development of Jiangsu Higher Education Institutions J.W. acknowledges the computational resources provided by SEU and National Supercomputing Center in Tianjin.

\section{Author contributions}

X.W. and Y.S. conceived and supervised the project. Z.Y. and R.X. carried out sample preparation, device fabrication, electrical measurements and data analysis. Y.P., Z.-Y.O, B.W., G.Z. and Y.W.Z. performed modelling of charge transport. Y.S., T.X. and L.S. performed TEM characterizations and analysis. Z.W. and J.W. performed density functional theory calculations. L.P. devised the MPS treatment method. X.W., Y.S., L.S and J.W. co-wrote the paper with all authors contributing to discussion and preparation of the manuscript.

\section{Additional information}

Supplementary Information accompanies this paper at http://www.nature.com/ naturecommunications

Competing financial interests: The authors declare no competing financial interests.

Reprints and permission information is available online at http://npg.nature.com/ reprintsandpermissions/

How to cite this article: Yu, Z. et al. Towards intrinsic charge transport in monolayer molybdenum disulfide by defect and interface engineering. Nat. Commun. 5:5290 doi: $10.1038 /$ ncomms6290 (2014) 